\title{
On a Generalized Laguerre Operational Matrix of Fractional Integration
}

\author{
A. H. Bhrawy, ${ }^{1,2}$ D. Baleanu, ${ }^{3,4,5}$ L. M. Assas, ${ }^{1,6}$ and J. A. Tenreiro Machado ${ }^{7}$ \\ ${ }^{1}$ Department of Mathematics, Faculty of Science, King Abdulaziz University, Jeddah 21589, Saudi Arabia \\ ${ }^{2}$ Department of Mathematics, Faculty of Science, Beni-Suef University, Beni-Suef 62511, Egypt \\ ${ }^{3}$ Department of Mathematics and Computer Sciences, Faculty of Arts and Sciences, Cankaya University, Eskisehir Yolu 29.km, \\ 06810 Yenimahalle Ankara, Turkey \\ ${ }^{4}$ Department of Chemical and Materials Engineering, Faculty of Engineering, King Abdulaziz University, Jeddah 21589, Saudi Arabia \\ ${ }^{5}$ Institute of Space Sciences, RO 76900, Magurele-Bucharest, Romania \\ ${ }^{6}$ Department of Mathematics, Faculty of Science, Umm Al-Qura University, Mecca 21955, Saudi Arabia \\ ${ }^{7}$ Department of Electrical Engineering, Polytechnic of Porto, Institute of Engineering, 4314200-072 Porto, Portugal
}

Correspondence should be addressed to D. Baleanu; dumitru@cankaya.edu.tr

Received 18 December 2012; Accepted 18 January 2013

Academic Editor: József Kázmér Tar

Copyright (C) 2013 A. H. Bhrawy et al. This is an open access article distributed under the Creative Commons Attribution License, which permits unrestricted use, distribution, and reproduction in any medium, provided the original work is properly cited.

\begin{abstract}
A new operational matrix of fractional integration of arbitrary order for generalized Laguerre polynomials is derived. The fractional integration is described in the Riemann-Liouville sense. This operational matrix is applied together with generalized Laguerre tau method for solving general linear multiterm fractional differential equations (FDEs). The method has the advantage of obtaining the solution in terms of the generalized Laguerre parameter. In addition, only a small dimension of generalized Laguerre operational matrix is needed to obtain a satisfactory result. Illustrative examples reveal that the proposed method is very effective and convenient for linear multiterm FDEs on a semi-infinite interval.
\end{abstract}

\section{Introduction}

The problems of FDEs arise in various areas of science and engineering. In particular, multiterm fractional differential equations have been used to model various types of viscoelastic damping (see, e.g., [1-13] and the references therein). In the last few decades both theory and numerical analysis of FDEs have received an increasing attention (see, e.g., $[1-4,14-$ 17] and references therein).

Spectral methods are a class of techniques used in applied mathematics and scientific computing to numerically solve some differential equations. The main idea is to write the solution of the differential equation as a sum of certain orthogonal polynomial and then obtain the coefficients in the sum in order to satisfy the differential equation. Due to high-order accuracy, spectral methods have gained increasing popularity for several decades, particularly in the field of computational fluid dynamics (see, e.g., [18-24] and the references therein).
The usual spectral methods are only available for bounded domains for solving FDEs; see [25-28]. However, it is also interesting to consider spectral methods for FDEs on the half line. Several authors developed the generalized Laguerre spectral method for the half line for ordinary, partial, and delay differential equations; see [29-31]. Recently, Saadatmandi and Dehghan [25] have proposed an operational Legendre-tau technique for the numerical solution of multiterm FDEs. The same technique based on operational matrix of Chebyshev polynomials has been used for the same problem (see [32]). In [33], Doha et al. derived the Jacobi operational matrix of fractional derivatives which applied together with spectral tau method for numerical solution of general linear multiterm fractional differential equations. Bhrawy et al. [27] used a quadrature shifted Legendre-tau method for treating multiterm linear FDEs with variable coefficients. More recently, Bhrawy and Alofi [34] proposed the operational 
Chebyshev matrix of fractional integration in the RiemannLiouville sense which was applied together with spectral tau method for solving linear FDEs.

The operational matrix of integer integration has been determined for several types of orthogonal polynomials, such as Chebyshev polynomials [35], Legendre polynomials [36], and Laguerre and Hermite [37]. Recently, Singh et al. [38] derived the Bernstein operational matrix of integration. Till now, and to the best of our knowledge, most of formulae corresponding to those mentioned previously are unknown and are traceless in the literature for fractional integration for generalized Laguerre polynomials in the Riemann-Liouville sense. This partially motivates our interest in operational matrix of fractional integration for generalized Laguerre polynomials. Another motivation is concerned with the direct solution techniques for solving the integrated forms of FDEs on the half line using generalized Laguerre tau method based on operational matrix of fractional integration in the Riemann-Liouville sense. Finally, the accuracy of the proposed algorithm is demonstrated by test problems.

The paper is organized as follows. In the next section, we introduce some necessary definitions. In Section 3 the generalized Laguerre operational matrix of fractional integration is derived. In Section 4 we develop the generalized Laguerre operational matrix of fractional integration for solving linear multiorder FDEs. In Section 5 the proposed method is applied to two examples.

\section{Some Basic Preliminaries}

The most used definition of fractional integration is due to Riemann-Liouville, which is defined as

$$
\begin{aligned}
J^{v} f(x)= & \frac{1}{\Gamma(v)} \int_{0}^{x}(x-t)^{\nu-1} f(t) d t \\
& v>0, \quad x>0, \quad \text { and } J^{0} f(x)=f(x) .
\end{aligned}
$$

The operator $J^{v}$ has the property:

$$
J^{v} x^{\beta}=\frac{\Gamma(\beta+1)}{\Gamma(\beta+1+v)} x^{\beta+v}
$$

The next equation defines the Riemann-Liouville fractional derivative of order $v$ :

$$
D^{v} f(x)=\frac{d^{m}}{d x^{m}}\left(J^{m-v} f(x)\right)
$$

where $m-1<v \leq m, m \in N$, and $m$ is the smallest integer greater than $v$.

If $m-1<v \leq m, m \in N$, then

$$
\begin{aligned}
& D^{v} J^{v} f(x)=f(x), \\
& J^{v} D^{v} f(x)=f(x)-\sum_{i=0}^{m-1} f^{(i)}\left(0^{+}\right) \frac{x^{i}}{i !}, \quad x>0 .
\end{aligned}
$$

Now, let $\Lambda=(0, \infty)$ and $w^{(\alpha)}(x)=x^{\alpha} e^{-x}$ be a weight function on $\Lambda$ in the usual sense. Define the following:

$$
\begin{aligned}
L_{w^{(\alpha)}}^{2}(\Lambda)= & \{v \mid v \text { is measurable on } \Lambda \text { and } \\
& \left.\|v\|_{w^{(\alpha)}}<\infty\right\},
\end{aligned}
$$

equipped with the following inner product and norm:

$$
\begin{aligned}
& (u, v)_{w^{(\alpha)}}=\int_{\Lambda} u(x) v(x) w^{(\alpha)}(x) d x, \\
& \|v\|_{w^{(\alpha)}}=(u, v)_{w^{(\alpha)}}^{1 / 2} .
\end{aligned}
$$

Next, let $L_{i}^{(\alpha)}(x)$ be the generalized Laguerre polynomials of degree $i$. We know from [39] that, for $\alpha>-1$,

$$
\begin{array}{r}
L_{i+1}^{(\alpha)}(x)=\frac{1}{i+1}\left[(2 i+\alpha+1-x) L_{i}^{(\alpha)}(x)-(i+\alpha) L_{i-1}^{(\alpha)}(x)\right], \\
i=1,2, \ldots,
\end{array}
$$

where $L_{0}^{(\alpha)}(x)=1$ and $L_{1}^{(\alpha)}(x)=1+\alpha-x$. The set of generalized Laguerre polynomials is the $L_{w^{(\alpha)}}^{2}(\Lambda)$-orthogonal system, namely,

$$
\int_{0}^{\infty} L_{j}^{(\alpha)}(x) L_{k}^{(\alpha)}(x) w^{(\alpha)}(x) d x=h_{k} \delta_{j k},
$$

where $\delta_{j k}$ is the Kronecher function and $h_{k}=(\Gamma(i+\alpha+1)) / i$ !.

The generalized Laguerre polynomials of degree $i$, on the interval $\Lambda$, are given by

$$
\begin{array}{r}
L_{i}^{(\alpha)}(x)=\sum_{k=0}^{i}(-1)^{k} \frac{\Gamma(i+\alpha+1)}{\Gamma(k+\alpha+1)(i-k) ! k !} x^{k} \\
i=0,1, \ldots
\end{array}
$$

The special value

$$
D^{q} L_{i}^{(\alpha)}(0)=(-1)^{q} \sum_{j=0}^{i-q} \frac{(i-j-1) !}{(q-1) !(i-j-q) !} L_{j}^{(\alpha)}(0), \quad i \geq q,
$$

where $L_{j}^{(\alpha)}(0)=(\Gamma(j+\alpha+1)) /(\Gamma(\alpha+1) j !)$, will be of important use later.

A function $u(x) \in L_{w^{(\alpha)}}^{2}(\Lambda)$ may be expressed in terms of generalized Laguerre polynomials as

$$
\begin{aligned}
u(x) & =\sum_{j=0}^{\infty} a_{j} L_{j}^{(\alpha)}(x), \\
a_{j} & =\frac{1}{h_{k}} \int_{0}^{\infty} u(x) L_{j}^{(\alpha)}(x) w^{(\alpha)}(x) d x, \quad j=0,1,2, \ldots
\end{aligned}
$$

In practice, only the first $(N+1)$ terms of generalized Laguerre polynomials are considered. Then we have

$$
u_{N}(x)=\sum_{j=0}^{N} a_{j} L_{j}^{(\alpha)}(x)=C^{T} \phi(x),
$$


where the generalized Laguerre coefficient vector $C$ and the generalized Laguerre vector $\phi(x)$ are given by

$$
\begin{aligned}
& C^{T}=\left[c_{0}, c_{1}, \ldots, c_{N}\right], \\
& \phi(x)=\left[L_{0}^{(\alpha)}(x), L_{1}^{(\alpha)}(x), \ldots, L_{N}^{(\alpha)}(x)\right]^{T} .
\end{aligned}
$$

If we define the $q$ times repeated integration of generalized Laguerre vector $\phi(x)$ by $J^{q} \phi(x)$, then (cf. Paraskevopoulos [36])

$$
J^{q} \phi(x) \simeq P^{(q)} \phi(x),
$$

where $q$ is an integer value and $\mathbf{P}^{(q)}$ is the operational matrix of integration of $\phi(x)$. For more details see [36].

\section{Generalized Laguerre Operational Matrix of Fractional Integration}

The main objective of this section is to derive an operational matrix of fractional integration for generalized Laguerre vector.

Theorem 1. Let $\phi(x)$ be the generalized Laguerre vector and $v>0$, then

$$
J^{v} \phi(x) \simeq \mathbf{P}^{(v)} \phi(x),
$$

where $\mathbf{P}^{(v)}$ is the $(N+1) \times(N+1)$ operational matrix of fractional integration of order $v$ in the Riemann-Liouville sense and is defined as follows:

$$
\begin{aligned}
& \mathbf{P}^{(v)} \\
& =\left(\begin{array}{ccccc}
\Theta_{v}(0,0) & \Omega_{v}(0,1) & \Theta_{v}(0,2) & \cdots & \Theta_{v}(0, N) \\
\Theta_{v}(1,0) & \Theta_{v}(1,1) & \Theta_{v}(1,2) & \cdots & \Theta_{v}(1, N) \\
\vdots & \vdots & \vdots & \cdots & \vdots \\
\Theta_{v}(i, 0) & \Theta_{v}(i, 1) & \Theta_{v}(i, 2) & \cdots & \Theta_{v}(i, N) \\
\vdots & \vdots & \vdots & \cdots & \vdots \\
\Theta_{v}(N, 0) & \Theta_{v}(N, 1) & \Theta_{v}(N, 2) & \cdots & \Theta_{v}(N, N)
\end{array}\right),
\end{aligned}
$$

where

$$
\begin{aligned}
& \Theta_{v}(i, j) \\
& =\sum_{k=0}^{i} \sum_{r=0}^{j} \frac{(-1)^{k+r} j ! \Gamma(i+\alpha+1) \Gamma(k+v+\alpha+r+1)}{(i-k) !(j-r) ! r ! \Gamma(k+v+1) \Gamma(k+\alpha+1) \Gamma(\alpha+r+1)} .
\end{aligned}
$$

Proof. Using the analytic form of the generalized Laguerre polynomials $L_{i}^{(\alpha)}(x)$ of degree $i(9)$ and (2), then

$$
\begin{aligned}
J^{v} L_{i}^{(\alpha)}(x)= & \sum_{k=0}^{i}(-1)^{k} \frac{\Gamma(i+\alpha+1)}{(i-k) ! k ! \Gamma(k+\alpha+1)} J^{v} x^{k} \\
= & \sum_{k=0}^{i}(-1)^{k} \frac{\Gamma(i+\alpha+1)}{(i-k) ! \Gamma(k+v+1) \Gamma(k+\alpha+1)} \\
& \times x^{k+v}, \quad i=0,1, \cdots, N .
\end{aligned}
$$

Now, approximate $x^{k+v}$ by $N+1$ terms of generalized Laguerre series, we have

$$
x^{k+v}=\sum_{j=0}^{N} c_{j} L_{j}^{(\alpha)}(x),
$$

where $c_{j}$ is given from (11) with $u(x)=x^{k+v}$; that is,

$$
c_{j}=\sum_{r=0}^{j}(-1)^{r} \frac{j ! \Gamma(k+v+\alpha+r+1)}{(j-r) ! r ! \Gamma(r+\alpha+1)}, \quad j=1,2, \ldots, N .
$$

In virtue of (18) and (19), we get

$$
J^{v} L_{i}^{(\alpha)}(x)=\sum_{j=0}^{N} \Theta_{v}(i, j) L_{j}^{(\alpha)}(x), \quad i=0,1, \ldots, N,
$$

where

$$
\begin{array}{r}
\Theta_{v}(i, j) \\
=\sum_{k=0}^{i} \sum_{r=0}^{j} \frac{(-1)^{k+r} j ! \Gamma(i+\alpha+1) \Gamma(k+v+\alpha+r+1)}{(i-k) !(j-r) ! r ! \Gamma(k+v+1) \Gamma(k+\alpha+1) \Gamma(\alpha+r+1)} \\
j=1,2, \ldots N .
\end{array}
$$

Accordingly, (21) can be written in a vector form as follows:

$$
\begin{aligned}
J^{v} L_{i}(x) \simeq & {\left[\Theta_{v}(i, 0), \Theta_{v}(i, 1), \Theta_{v}(i, 2), \cdots,\right.} \\
& \left.\Theta_{v}(i, N)\right] \phi(x), \quad i=0,1, \ldots, N .
\end{aligned}
$$

Equation (23) leads to the desired result.

\section{Generalized Laguerre Tau Method Based on Operational Matrix}

In this section, the generalized Laguerre tau method based on operational matrix is proposed to numerically solve FDEs. In order to show the fundamental importance of generalized Laguerre operational matrix of fractional integration, we adopt it for solving the following multiorder FDE:

$$
\begin{array}{r}
D^{v} u(x)=\sum_{i=1}^{k} \gamma_{j} D^{\beta_{i}} u(x)+\gamma_{k+1} u(x)+f(x), \\
\operatorname{in} \Lambda=(0, \infty),
\end{array}
$$

with initial conditions

$$
u^{(i)}(0)=d_{i}, \quad i=0, \ldots, m-1,
$$

where $\gamma_{i}(i=1, \ldots, k+1)$ are real constant coefficients, $m-1<$ $v \leq m, 0<\beta_{1}<\beta_{2}<\ldots<\beta_{k}<v$, and $g(x)$ is a given source function.

The proposed technique, based on the FDE (24), is converted to a fully integrated form via fractional integration 
in the Riemann-Liouville sense. Subsequently, the integrated form equations are approximated by representing them as linear combinations of generalized Laguerre polynomials. Finally, the integrated form equation is converted to an algebraic equation by introducing the operational matrix of fractional integration of the generalized Laguerre polynomials.

If we apply the Riemann-Liouville integral of order $v$ on (24), after making use of (4), we get the integrated form of (24), namely,

$$
\begin{aligned}
& u(x)-\sum_{j=0}^{m-1} u^{(j)}\left(0^{+}\right) \frac{x^{j}}{j !} \\
&= \sum_{i=1}^{k} \gamma_{i} J^{v-\beta_{i}}\left[u(x)-\sum_{j=0}^{m_{i}-1} u^{(j)}\left(0^{+}\right) \frac{x^{j}}{j !}\right] \\
&+\gamma_{k+1} J^{v} u(x)+J^{v} f(x), \\
& u^{(i)}(0)=d_{i}, \quad i=0, \ldots, m-1,
\end{aligned}
$$

where $m_{i}-1<\beta_{i} \leq m_{i}, m_{i} \in N$, implies that

$$
\begin{aligned}
& u(x)=\sum_{i=1}^{k} \gamma_{i} J^{v-\beta_{i}} u(x)+\gamma_{k+1} J^{v} u(x)+g(x), \\
& u^{(i)}(0)=d_{i}, \quad i=0, \ldots, m-1,
\end{aligned}
$$

where

$$
g(x)=J^{v} f(x)+\sum_{j=0}^{m-1} d_{j} \frac{x^{j}}{j !}+\sum_{i=1}^{k} \gamma_{i} J^{\nu-\beta_{i}}\left(\sum_{j=0}^{m_{i}-1} d_{j} \frac{x^{j}}{j !}\right) .
$$

In order to use the tau method with Laquerre operational matrix for solving the fully integrated problem (27) with initial conditions (25), we approximate $u(x)$ and $g(x)$ by the Laguerre polynomials:

$$
\begin{aligned}
& u_{N}(x) \simeq \sum_{i=0}^{N} c_{i} L_{i}^{(\alpha)}(x)=C^{T} \phi(x), \\
& g(x) \simeq \sum_{i=0}^{N} g_{i} L_{i}^{(\alpha)}(x)=G^{T} \phi(x),
\end{aligned}
$$

where the vector $G=\left[g_{0}, \ldots, g_{N}\right]^{T}$ is given but $C=\left[c_{0}, \ldots\right.$, $\left.c_{N}\right]^{T}$ is an unknown vector.

After making use of Theorem 1 (relation (15)) the Riemann-Liouville integral of orders $v$ and $\left(\nu-\beta_{j}\right)$ of the approximate solution (29) can be written as

$$
\begin{array}{r}
J^{v} u_{N}(x) \simeq C^{T} J^{v} \phi(x) \simeq C^{T} \mathbf{P}^{(v)} \phi(x), \\
J^{v-\beta_{j}} u_{N}(x) \simeq C^{T} J^{\nu-\beta_{j}} \phi(x) \simeq C^{T} \mathbf{P}^{\left(v-\beta_{j}\right)} \phi(x), \\
j=1, \ldots, k,
\end{array}
$$

respectively, where $\mathbf{P}^{(v)}$ is the $(N+1) \times(N+1)$ operational matrix of fractional integration of order $v$. Employing (29)(32) the residual $R_{N}(x)$ for (27) can be written as

$$
R_{N}(x)=\left(C^{T}-C^{T} \sum_{j=1}^{k} \gamma_{j} \mathbf{P}^{\left(v-\beta_{j}\right)}-\gamma_{k+1} C^{T} \mathbf{P}^{(v)}-G^{T}\right) \phi(x) .
$$

As in a typical tau method, we generate $N-m+1$ linear algebraic equations by applying

$$
\begin{array}{r}
\left\langle R_{N}(x), L_{j}^{(\alpha)}(x)\right\rangle=\int_{0}^{\infty} R_{N}(x) w^{(\alpha)}(x) L_{j}^{(\alpha)}(x) d x=0, \\
j=0,1, \ldots, N-m .
\end{array}
$$

Also by substituting Eqs. (11) and (29) in Eq (25), we get

$$
u^{(i)}(0)=C^{T} \mathbf{D}^{(i)} \phi(0)=d_{i}, \quad i=0,1, \ldots, m-1 .
$$

Equations (34) and (35) generate $N-m+1$ and $m$ set of linear equations, respectively.

These linear equations can be solved for unknown coefficients of the vector $C$. Consequently, $u_{N}(x)$ given in (29) can be calculated, which leads to the solution of (24) with the initial conditions (25).

\section{Illustrative Examples}

To illustrate the effectiveness of the proposed method in the present paper, two test examples are carried out in this section. The results obtained by the present methods reveal that the present method is very effective and convenient for linear FDEs on the half line.

\section{Example 2. Consider the FDE}

$$
\begin{aligned}
& D^{2} u(x)+D^{1 / 2} u(x)+u(x) \\
& =x^{2}+2+\frac{2.6666666667}{\Gamma(0.5)} x^{1.5}, \\
& u(0)=0, \quad u^{\prime}(0)=0, \quad x \in \Lambda,
\end{aligned}
$$

whose exact solution is given by $u(x)=x^{2}$.

If we apply the technique described in Section 4 with $N=$ 2 , then the approximate solution can be written as

$$
\begin{aligned}
u_{N}(x)= & \sum_{i=0}^{2} c_{i} L_{i}^{(\alpha)}(x)=C^{T} \phi(x) \\
P^{(2)} & =\left(\begin{array}{lll}
\Theta_{2}(0,0) & \Theta_{2}(0,1) & \Theta_{2}(0,2) \\
\Theta_{2}(1,0) & \Theta_{2}(1,1) & \Theta_{2}(1,2) \\
\Theta_{2}(2,0) & \Theta_{2}(2,1) & \Theta_{2}(2,2)
\end{array}\right) \\
P^{(3 / 2)} & =\left(\begin{array}{lll}
\Theta_{3 / 2}(0,0) & \Theta_{3 / 2}(0,1) & \Theta_{3 / 2}(0,2) \\
\Theta_{3 / 2}(1,0) & \Theta_{3 / 2}(1,1) & \Theta_{3 / 2}(1,2) \\
\Theta_{3 / 2}(2,0) & \Theta_{3 / 2}(2,1) & \Theta_{3 / 2}(2,2)
\end{array}\right), \\
G & =\left(\begin{array}{l}
g_{0} \\
g_{1} \\
g_{2}
\end{array}\right) .
\end{aligned}
$$


TABLE 1: $c_{0}, c_{1}$, and $c_{2}$, for different values of $\alpha$ for Example 2 .

\begin{tabular}{lcll}
\hline$\alpha$ & $c_{0}$ & $c_{1}$ & $c_{2}$ \\
\hline-0.5 & 0.75 & -3 & 2 \\
0 & 2 & -4 & 2 \\
0.5 & 3.75 & -5 & 2 \\
1 & 6 & -6 & 2 \\
2 & 12 & -8 & 2 \\
3 & 20 & -10 & 2 \\
\hline
\end{tabular}

Using (34) we obtain

$$
\begin{aligned}
& \left(\Theta_{3 / 2}(0,2)+\Theta_{2}(0,2)\right) c_{0}+\left(\Theta_{3 / 2}(1,2)+\Theta_{2}(1,2)\right) c_{1} \\
& +\left(1+\Theta_{3 / 2}(2,2)+\Theta_{2}(2,2)\right) c_{2}+g_{2}=0 .
\end{aligned}
$$

Now, by applying (35), we have

$$
\begin{gathered}
c_{0}+(\alpha+1) c_{1}+\frac{(\alpha+1)(\alpha+2)}{2} c_{2}=0 . \\
-c_{1}-(\alpha+2) c_{2}=0 .
\end{gathered}
$$

Finally by solving (38)-(40), we have the 3 unknown coefficients with various choices of $\alpha$ given in Table 1 . Then, we get

$$
c_{0}=\alpha^{2}+3 \alpha+2, \quad c_{1}=-2 \alpha-4, \quad c_{2}=2 .
$$

Thus we can write

$$
u(x)=\left(\begin{array}{lll}
c_{0} & c_{1}, & c_{2}
\end{array}\right)\left(\begin{array}{l}
L_{0}^{(\alpha)}(x) \\
L_{1}^{(\alpha)}(x) \\
L_{2}^{(\alpha)}(x)
\end{array}\right)=x^{2}
$$

which is the exact solution.

Example 3. As the first example, we consider the following fractional initial value problem:

$$
\begin{array}{r}
D^{3 / 2} u(x)+3 u(x)=3 x^{3}+\frac{8}{\Gamma(0.5)} x^{1.5}, \\
u(0)=0, \quad u^{\prime}(0)=0, \quad x \in \Lambda,
\end{array}
$$

whose exact solution is given by $u(x)=x^{3}$.

If we apply the technique described in Section 4 with $N=$ 3 , then the approximate solution can be written as

$$
\begin{gathered}
u_{N}(x)=\sum_{i=0}^{3} c_{i} L_{i}^{(\alpha)}(x)=C^{T} \phi(x) \\
P^{(3 / 2)}=\left(\begin{array}{llll}
\Theta_{3 / 2}(0,0) & \Theta_{3 / 2}(0,1) & \Theta_{3 / 2}(0,2) & \Theta_{3 / 2}(0,3) \\
\Theta_{3 / 2}(1,0) & \Theta_{3 / 2}(1,1) & \Theta_{3 / 2}(1,2) & \Theta_{3 / 2}(1,3) \\
\Theta_{3 / 2}(2,0) & \Theta_{3 / 2}(2,1) & \Theta_{3 / 2}(2,2) & \Theta_{3 / 2}(2,3) \\
\Theta_{3 / 2}(3,0) & \Theta_{3 / 2}(3,1) & \Theta_{3 / 2}(3,2) & \Theta_{3 / 2}(3,3)
\end{array}\right), \\
G=\left(\begin{array}{l}
g_{0} \\
g_{1} \\
g_{2} \\
g_{3}
\end{array}\right) .
\end{gathered}
$$

TABLE 2: $c_{0}, c_{1}, c_{2}$ and $c_{3}$ for different values of $\alpha$ for Example 3.

\begin{tabular}{lccll}
\hline$\alpha$ & $c_{0}$ & $c_{1}$ & $c_{2}$ & $c_{3}$ \\
\hline-0.5 & $15 / 8$ & $-45 / 4$ & 15 & -6 \\
0 & 6 & -18 & 18 & -6 \\
0.5 & $105 / 8$ & $-105 / 4$ & 21 & -6 \\
1 & 24 & -36 & 24 & -6 \\
2 & 60 & -60 & 30 & -6 \\
3 & 120 & -90 & 36 & -6 \\
\hline
\end{tabular}

Using (34) we obtain

$$
\begin{aligned}
& 3 \Theta_{3 / 2}(0,2) c_{0}+3 \Theta_{3 / 2}(1,2) c_{1} \\
& +\left(1+3 \Theta_{3 / 2}(2,2)\right) c_{2}+3 \Theta_{3 / 2}(3,2) c_{3}+g_{2}=0 \\
& 3 \Theta_{3 / 2}(0,3) c_{0}+3 \Theta_{3 / 2}(1,3) c_{1} \\
& \quad+3 \Theta_{3 / 2}(2,3) c_{2}+\left(1+3 \Theta_{3 / 2}(3,3)\right) c_{3}+g_{3}=0 .
\end{aligned}
$$

Now, applying (35) we get

$$
\begin{aligned}
& C^{T} \phi(0)=c_{0}+(\alpha+1) c_{1} \\
& \quad+\frac{(\alpha+1)(\alpha+2)}{2} c_{2}+\frac{(\alpha+1)(\alpha+2)(\alpha+3)}{6} c_{3}=0, \\
& C^{T} D^{(1)} \phi(0)
\end{aligned}
$$

$$
=-c_{1}-(\alpha+2) c_{2}-\frac{(\alpha+3)(\alpha+2)}{2} c_{3}=0 .
$$

By solving the linear system (45)-(49) we have the 4 unknown coefficients with various choices of $\alpha$ in Table 2 , and we get

$$
\begin{aligned}
& c_{0}=\alpha^{3}+6 \alpha+11 \alpha+6, \\
& c_{1}=-3 \alpha^{2}-15 \alpha-18, \\
& c_{2}=6 \alpha+18, \\
& c_{3}=-6 .
\end{aligned}
$$

Thereby we can write

$$
u_{N}(x)=\sum_{i=0}^{3} c_{i} L_{i}^{(\alpha)}(x)=x^{3} .
$$

Numerical results will not be presented since the exact solution is obtained.

Example 4. Consider the following equation:

$$
\begin{aligned}
& D^{2} u(x)-2 D u(x)+D^{1 / 2} u(x)+u(x) \\
& =x^{7}+\frac{2048}{429 \sqrt{\pi}} x^{6.5}-14 x^{6}+42 x^{5}-x^{2}-\frac{8}{3 \sqrt{\pi}} x^{1.5}+4 x-2, \\
& u(0)=0, \quad u^{\prime}(0)=0, \quad x \in \Lambda,
\end{aligned}
$$

whose exact solution is given by $u(x)=x^{7}-x^{2}$. 
Now, we can apply the technique described in Examples 2 and 3, with $\alpha=0$ and $N=7$, then we have

$$
\begin{array}{ll}
c_{0}=5038, & c_{1}=-35276, \\
c_{2}=105838, & c_{3}=-176400, \\
c_{4}=176400, & c_{5}=-105840, \\
c_{6}=35280, & c_{7}=-5040 .
\end{array}
$$

Thus we can write

$$
u_{N}(x)=\sum_{i=0}^{7} c_{i} L_{i}(x)=x^{7}-x^{2},
$$

which is the exact solution.

\section{Conclusions}

In this paper, we have presented the operational matrix of fractional integration of the generalized Laguerre polynomials, and, as an important application, we describe how to use the operational tau technique to numerically solve the FDEs. The basic idea of this technique is as follows.

(i) The FDE is converted to a fully integrated form via multiple integration in the Riemann-Liouville sense.

(ii) Subsequently, the various signals involved in the integrated form equation are approximated by representing them as linear combinations of generalized Laguerre polynomials.

(iii) Finally, the integrated form equation is converted into an algebraic equation by introducing the operational matrix of fractional integration of the generalized Laguerre polynomials.

To the best of our knowledge, the presented theoretical formula for generalized Laguerre is completely new, and we do believe that this formula may be used to solve some other kinds of fractional-order initial value problems on a semiinfinite interval.

\section{References}

[1] I. Podlubny, Fractional Differential Equations, vol. 198 of Mathematics in Science and Engineering, Academic Press, San Diego, Calif, USA, 1999.

[2] S. Das, Functional Fractional Calculus for System Identification and Controls, Springer, Berlin, Germany, 2008.

[3] A. A. Kilbas, H. M. Srivastava, and J. J. Trujillo, Theory and Applications of Fractional Differential Equations, vol. 204 of North-Holland Mathematics Studies, Elsevier Science B.V., Amsterdam, The Netherlands, 2006.

[4] S. Das, K. Vishal, P. K. Gupta, and A. Yildirim, "An approximate analytical solution of time-fractional telegraph equation," Applied Mathematics and Computation, vol. 217, no. 18, pp. 7405-7411, 2011.

[5] D. Baleanu, K. Diethelm, E. Scalas, and J. J. Trujillo, Fractional Calculus Models and Numerical Methods, vol. 3 of Series on Complexity, Nonlinearity and Chaos, World Scientific, Hackensack, NJ, USA, 2012.
[6] R. Hilfer, Ed., Applications of Fractional Calculus in Physics, World Scientific, River Edge, NJ, USA, 2000.

[7] D. Baleanu, J. A. T. Machado, and A. C. J. Luo, Eds., Fractional Dynamics and Control, Springer, New York, NY, USA, 2012.

[8] J. T. Machado, V. Kiryakova, and F. Mainardi, "Recent history of fractional calculus," Communications in Nonlinear Science and Numerical Simulation, vol. 16, no. 3, pp. 1140-1153, 2011.

[9] C. M. A. Pinto, "Stability of quadruped robots' trajectories subjected to discrete perturbations," Nonlinear Dynamics, vol. 70, no. 3, pp. 2089-2094, 2012.

[10] H. Jafari, H. Tajadodi, and D. Baleanu, "A modified variational iteration method for solving fractional Riccati differential equation by Adomian polynomials," Fractional Calculus and Applied Analysis, vol. 16, no. 1, pp. 109-122, 2013.

[11] C. M. Ionescu and R. De Keyser, "Relations between fractionalorder model parameters and lung pathology in chronic obstructive pulmonary disease," IEEE Transactions on Biomedical Engineering, vol. 56, no. 4, pp. 978-987, 2009.

[12] C. M. Ionescu, P. Segers, and R. De Keyser, "Mechanical properties of the respiratory system derived from morphologic insight," IEEE Transactions on Biomedical Engineering, vol. 56, no. 4, pp. 949-959, 2009.

[13] S. Bhalekar, V. Daftardar-Gejji, D. Baleanu, and R. Magin, "Transient chaos in fractional Bloch equations," Computers \& Mathematics with Applications, vol. 64, no. 10, pp. 3367-3376, 2012.

[14] S. Esmaeili, M. Shamsi, and Y. Luchko, "Numerical solution of fractional differential equations with a collocation method based on Müntz polynomials," Computers \& Mathematics with Applications, vol. 62, no. 3, pp. 918-929, 2011.

[15] Z. Odibat, S. Momani, and V. S. Erturk, "Generalized differential transform method: application to differential equations of fractional order," Applied Mathematics and Computation, vol. 197, no. 2, pp. 467-477, 2008.

[16] D. Băleanu, O. G. Mustafa, and R. P. Agarwal, "An existence result for a superlinear fractional differential equation," Applied Mathematics Letters, vol. 23, no. 9, pp. 1129-1132, 2010.

[17] D. Băleanu, O. G. Mustafa, and R. P. Agarwal, "On the solution set for a class of sequential fractional differential equations," Journal of Physics A, vol. 43, no. 38, p. 385209, 7, 2010.

[18] E. H. Doha and W. M. Abd-Elhameed, "Efficient solutions of multidimensional sixth-order boundary value problems using symmetric generalized Jacobi-Galerkin method," Abstract and Applied Analysis, vol. 2012, Article ID 749370, 19 pages, 2012.

[19] A. H. Bhrawy and Al-Shomrani, "A Jacobi dual-Petrov Galerkin-Jacobi collocation method for solving Korteweg-de Vries equations," Abstract and Applied Analysis, vol. 2012, Article ID 418943, 16 pages, 2012.

[20] A. H. Bhrawy and M. A. Alghamdi, "Numerical solutions of odd order linear and nonlinear initial value problems using shifted Jacobi spectral approximations," Abstract and Applied Analysis, vol. 2012, Article ID 364360, 25 pages, 2012.

[21] E. H. Doha, A. H. Bhrawy, and R. M. Hafez, "On shifted Jacobi spectral method for high-order multi-point boundary value problems," Communications in Nonlinear Science and Numerical Simulation, vol. 17, no. 10, pp. 3802-3810, 2012.

[22] A. H. Bhrawy, A. S. Alofi, and S. I. El-Soubhy, "Spectral shifted Jacobi tau and collocation methods for solving fifth-order boundary value problems," Abstract and Applied Analysis, vol. 2011, Article ID 823273, 14 pages, 2011. 
[23] E. H. Doha, A. H. Bhrawy, and R. M. Hafez, "A Jacobi dualPetrov-Galerkin method for solving some odd-order ordinary differential equations," Abstract and Applied Analysis, vol. 2011, Article ID 947230, 21 pages, 2011.

[24] A. H. Bhrawy and W. M. Abd-Elhameed, "New algorithm for the numerical solutions of nonlinear third-order differential equations using Jacobi-Gauss collocation method," Mathematical Problems in Engineering, vol. 2011, Article ID 837218, 14 pages, 2011.

[25] A. Saadatmandi and M. Dehghan, "A new operational matrix for solving fractional-order differential equations," Computers \& Mathematics with Applications, vol. 59, no. 3, pp. 1326-1336, 2010.

[26] E. H. Doha, A. H. Bhrawy, and S. S. Ezz-Eldien, "Efficient Chebyshev spectral methods for solving multi-term fractional orders differential equations," Applied Mathematical Modelling, vol. 35, no. 12, pp. 5662-5672, 2011.

[27] A. H. Bhrawy, A. S. Alofi, and S. S. Ezz-Eldien, "A quadrature tau method for fractional differential equations with variable coefficients," Applied Mathematics Letters, vol. 24, no. 12, pp. 2146-2152, 2011.

[28] A. H. Bhrawy and M. M. Al-Shomrani, "A shifted Legendre spectral method for fractional-order multi-point boundary value problems," Advances in Differential Equations, 2012.

[29] B.-Y. Guo and L.-L. Wang, "Modified Laguerre pseudospectral method refined by multidomain Legendre pseudospectral approximation," Journal of Computational and Applied Mathematics, vol. 190, no. 1-2, pp. 304-324, 2006.

[30] D. Funaro, "Estimates of Laguerre spectral projectors in Sobolev spaces," in Orthogonal Polynomials and Their Applications (Erice, 1990), C. Brezinski, L. Gori, and A. Ronveaux, Eds., vol. 9 of IMACS Ann. Comput. Appl. Math., pp. 263-266, Baltzer, Basel, Switzerland, 1991.

[31] M. Gülsu, B. Gürbüz, Y. Öztürk, and M. Sezer, "Laguerre polynomial approach for solving linear delay difference equations," Applied Mathematics and Computation, vol. 217, no. 15, pp. 6765-6776, 2011

[32] E. H. Doha, A. H. Bhrawy, and S. S. Ezz-Eldien, "A Chebyshev spectral method based on operational matrix for initial and boundary value problems of fractional order," Computers \& Mathematics with Applications, vol. 62, no. 5, pp. 2364-2373, 2011.

[33] E. H. Doha, A. H. Bhrawy, and S. S. Ezz-Eldien, "A new Jacobi operational matrix: an application for solving fractional differential equations," Applied Mathematical Modelling, vol. 36, no. 10, pp. 4931-4943, 2012.

[34] A. H. Bhrawy and A. S. Alofi, "The operational matrix of fractional integration for shifted Chebyshev polynomials," Applied Mathematics Letters, vol. 26, no. 1, pp. 25-31, 2013.

[35] P. N. Paraskevopoulos, "Chebyshev series approach to system identification, analysis and optimal control," Journal of the Franklin Institute, vol. 316, no. 2, pp. 135-157, 1983.

[36] P. N. Paraskevopoulos, "Legendre series approach to identification and analysis of linear systems," IEEE Transactions on Automatic Control, vol. 30, no. 6, pp. 585-589, 1985.

[37] E. H. Doha, H. M. Ahmed, and S. I. El-Soubhy, "Explicit formulae for the coefficients of integrated expansions of Laguerre and Hermite polynomials and their integrals," Integral Transforms and Special Functions, vol. 20, no. 7-8, pp. 491-503, 2009.

[38] A. K. Singh, V. K. Singh, and O. P. Singh, "The Bernstein operational matrix of integration," Applied Mathematical Sciences, vol. 3, no. 49-52, pp. 2427-2436, 2009.
[39] G. Szegö, Orthogonal Polynomials, American Mathematical Society, Providence, RI, USA, 1959. 


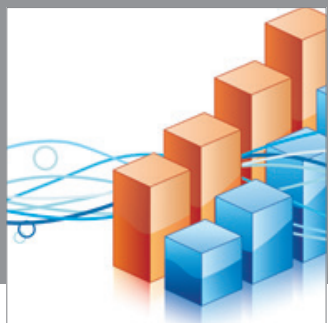

Advances in

Operations Research

mansans

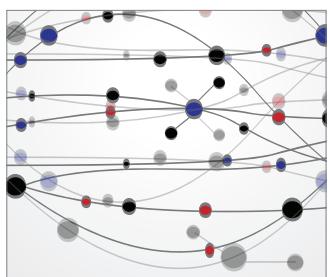

The Scientific World Journal
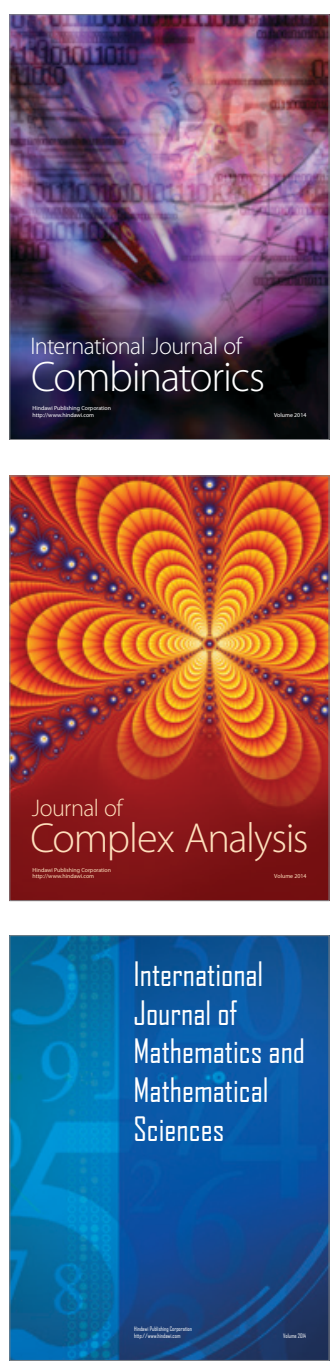
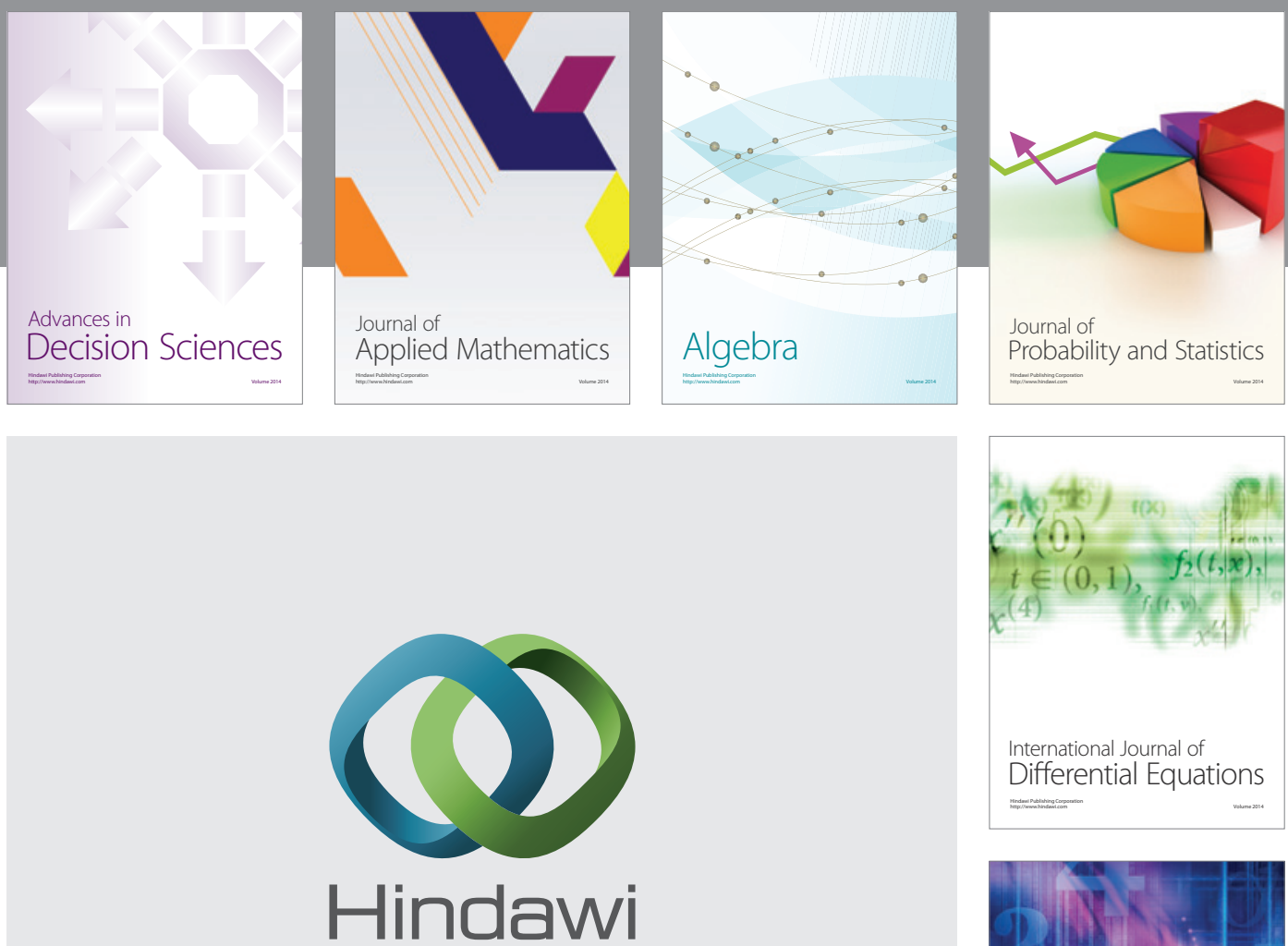

Submit your manuscripts at http://www.hindawi.com
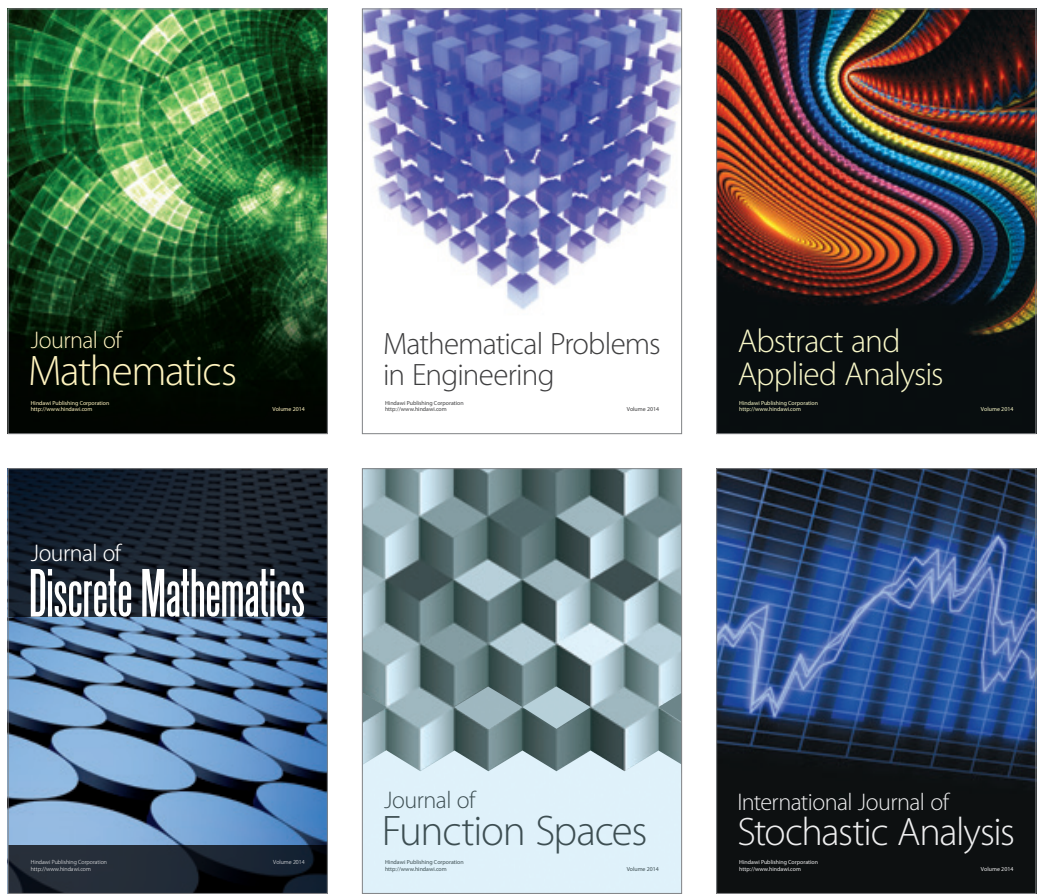

Journal of

Function Spaces

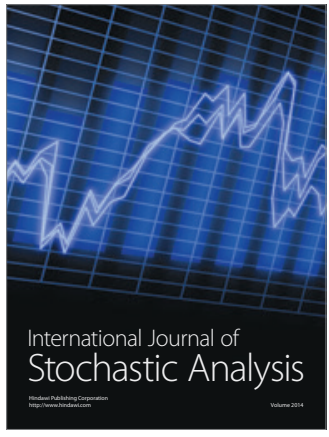

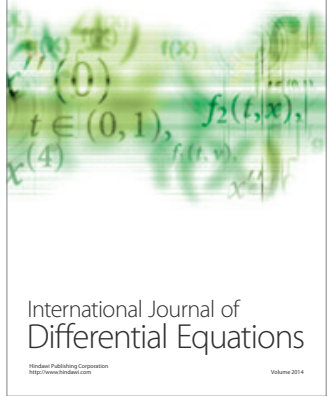
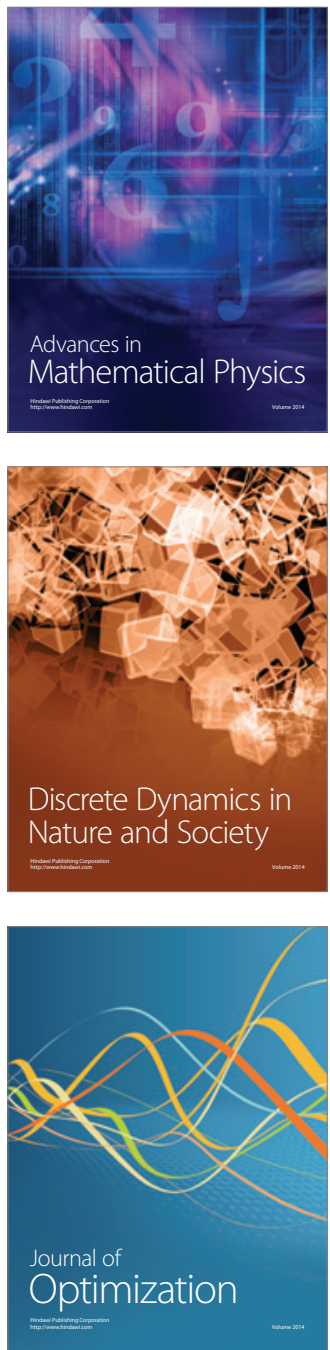